\title{
A New Cell Balancing Architecture for Li-ion Battery Packs Based on Cell Redundancy
}

\author{
Antonio Manenti*, Andrea Abba*, Angelo Geraci*, Sergio Savaresi* \\ *Politecnico di Milano, Department of Electrical Engineering, Milan (Italy) \\ manenti@elet.polimi.it \\ abba@elet.polimi.it \\ geraci@elet.polimi.it \\ savaresi@elet.polimi.it
}

\begin{abstract}
Li-ion batteries are promoting the development of more and more performing electric vehicles (EVs) and hybrid EVs (HEVs). Thanks to their high energy density and increased current capabilities which permit to use a relative light and space saving battery pack- these batteries are replacing almost all older battery chemistries in vehicle applications. Despite the advantages these cells provide, before use them in a new design, several drawbacks about safety aspects have to be considered. In fact, Li-ion battery packs require, more than other chemistry base packs, special control systems to maintain the operative conditions in a safe area and to guarantee the best performances in terms of efficiency and pack life time. One of the most challenging problems that have to be tackled by the mentioned control system is the voltage unbalance. This problem arises when more cells are connected in series with the purpose to increase the battery pack voltage. In this paper a new cell balancing architecture based on cell redundancy is proposed. This solution is especially suited to be implemented in vehicle battery management system (BMS) where cost and space reduction are the main requirements.
\end{abstract}

\section{INTRODUCTION}

The priority of electric vehicles as a primary factor of sustainable mobility is widely recognized worldwide also in the low-power two-wheels segment, i.e. scooters and bikes.

Lithium-ion (Li-ion) batteries, thanks to their high energy density, are commonly used for portable utilities and industry applications. Since voltage of a single cell is generally too low, for many high power applications such as electric vehicles (EVs), hybrid EVs (HEVs) plug-in HEVs (PHEVs), electric scooters, bikes, wheelchairs, and so on, string cells are often used. In this configuration, since the voltage of each cell can slight vary with respect to the others, the problem of cell imbalance can arise. In fact, during the charge phase, due to different internal resistance, chemical variations and temperature gradient along the pack, can happen that some cells reach the full charge condition before others, and, most important, before the entire pack charge is completed. This situation, if unmanaged, could determine overcharge of some cells and undercharge of some others. In the same way, during the discharge phase, some cells can experience over-discharge whereas others could still store available energy.

Moreover, the repetition of charge/discharge cycles increases the voltage imbalance, which is particularly detrimental in EV applications because of charge/discharge dynamics. In fact, unlike standard applications where almost complete charge/discharge phases follow one another, in EV use (except for PEHV) charge/discharge phases are partial and interleaved. This is due to the fact that discharge occurs during acceleration and drive whereas charge during regenerative braking (A. Emadi 2008). This voltage imbalance, although can be detrimental regardless the battery chemistry, is particularly harmful for Li-ion battery. In fact, it is well known that Li-ion batteries, like other chemistries, have both maximum and minimum voltage limit, which depend on specific cell. In this case however, exceeding these limits could entail severe safety problems since these batteries have no natural gassing mechanism to release an excess of energy with consequent explosion risk (H.V. Venkatasetty 2002). For this reason, a charge/discharge equalization procedure able to considerably improve the maintenance in time of cells efficiency is fundamental in case of vehicle Liion battery packs.

Many architectures have been proposed in order to prevent cell imbalance during charge/discharge of series-connected battery packs (Affanni October, 2005), (N. H. Kutkut 1999), (Ferdowsi 2005), (C. S.Moo 2003) and (J. Chatzakis 2003). Each of these solutions aims to a specific goal such as cost reduction, space saving (P. Casasso 2003), robustness, and so on. We present a new balancing approach that is suited for battery packs made up of a maximum $10 \mathrm{Li}$-ion cells for lowmedium power applications (in the range from $100 \mathrm{~W}$ to $500 \mathrm{~W}$ ). This solution can be oriented to light electric vehicles (i.e. electric bicycles), which usually need low-medium voltage $(24 \mathrm{~V}-36 \mathrm{~V})$ and current $(10 \mathrm{~A})$ associated to a simple, inexpensive and space-saving layout. In agreement with classification proposed in (Hong-Sun Park 2009), we focused on non-dissipative bidirectional equalizers. First of all, this means that the proposed solution doesn't use extra-power to prevent imbalance, thereby increasing the overall efficiency. In addition, it continues to work during both charge and discharge operation phases, improving balancing effectiveness. The proposed strategy is based on the availability of an additional cell that can be continuously disconnected from the load. In this way, the bypassed cell is 
isolated and can be optimally balanced while the remainder of the pack continues to provide energy to the load. It has to be notice that while pack works -charge or discharge- at least one cell is always disconnected from the string.

There are three main features of the proposed architecture. First, balancing is active both in charge and discharge phase. Second, the system layout is considerably space saving, which is a primary task for two-wheel vehicle applications often subjected to hard space constraints. Third, the solution offers an intrinsic hardiness to a cell fault. In fact, if the pack should contain a cell with a severe fault, the control system can identify and bypass it permanently.

\section{ARCHITECTURE}

The balancing system has been designed bearing in mind a general-purpose application, both in terms of cell type and operating conditions. So, standard Lithium-Ion cells with the following features have used as reference:

- $4.2 \mathrm{~V}$ of full charge voltage

- $\quad 4400 \mathrm{mAh}$ of capacity

- 10 A of maximum continuous current load

- 3 A of maximum charge current

All the system has been designed to manage a 7 cells battery pack. Consequently, it is able to manage a pack with a $25 \mathrm{~V}$ of open circuit voltage 10A of continuous load and nominal power of about $250 \mathrm{~W}$. Figure 1 shows the typical discharge profile of the used cell.

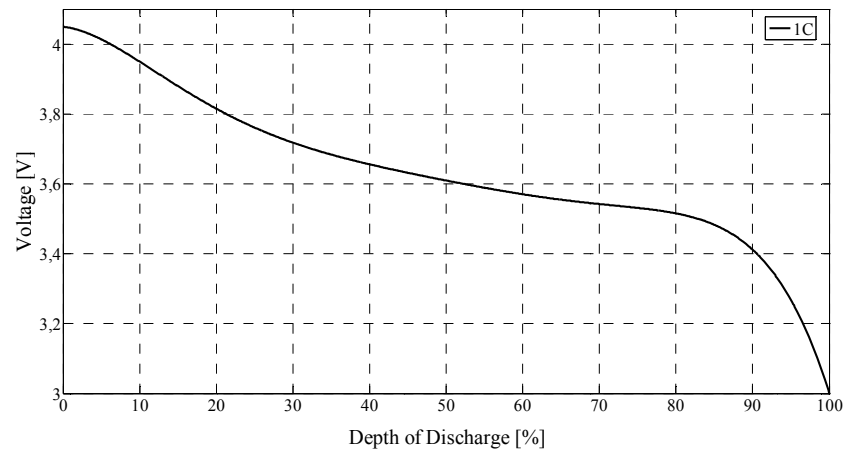

Figure 1: Typical discharge profile of a Lithium-Cobalt cell at $1 \mathrm{C}$.

The block diagram of the experimental setup is shown in Figure 2. It is constituted by three main stages: the switch network -first responsible of the performance of the entire system-, the data acquisition block, and the control unit. In the following the switch network will be deeply analyzed whereas a detailed explanation of the other two blocks is beyond the scope of this paper.

\section{A. Switch network}

The switch network plays a crucial role since it is critical both from a safety and efficiency points of view. Even if at a first glance the structure and the operation modality of the circuit are simple (Figure 3), the implementation revealed several tricky issues both in layout and management algorithm.

In details:
- Since switches are connected in series on the load current path, their resistance directly impacts on performances (in terms of efficiency) of the system.

- Switches have to be able to interrupt current flow in both directions. In fact, the system must perform cell balancing both in charge phase, i.e. current flowing from external terminal to cells, and in discharge phase, i.e. current flowing from cells to external load.

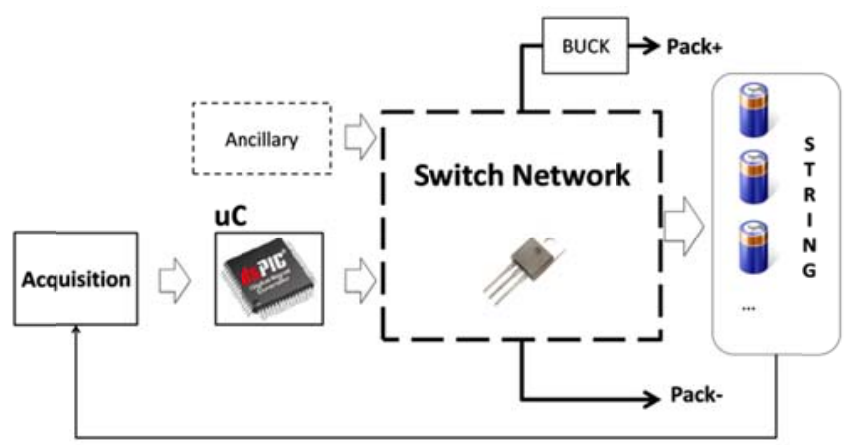

Figure 2: Block diagram of the proposed BMS architecture. Cores of the system are the data acquisition block (“Acquisition"), the control unit (the microcontroller "uC"), and the "Switch Network".

- Topic of the implementation is to minimize the number of components, which also prevents the necessity to use specific drivers of switches.

- The system has to be safe in every operative condition, e.g. even if a single cell be physically taken out from pack or microcontroller stops for a while.

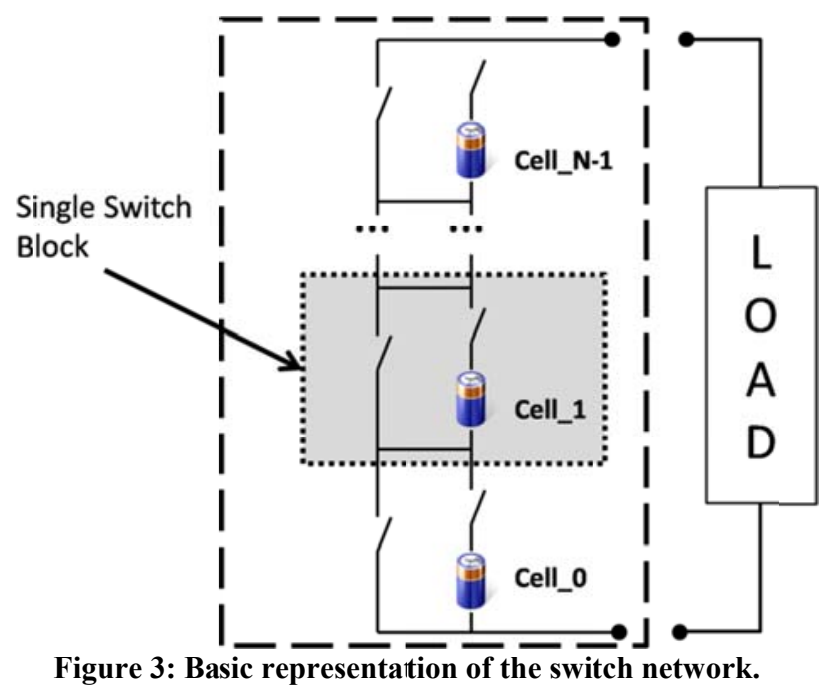

All these constraints make design of the stage critical both from layout and component choice points of view.

A simplified final schematic of the realized single switch between two adjacent cells is shown in Figure 4. Each cell has two switches, one series connected to the cell and the other one bypassing the cascade cell-switch. The single switch is constituted by two MOSFETs, in particular by N-channel MOSFETs (NMOS) for the series switch and by P-channel MOSFETs (PMOS) for the bypass switch. In this way, during 
normal operation 6 cells are connected by NMOS switches and one cell is bypassed through a PMOS switch. This represents a significant advantage due to the lower onresistance of NMOS compared with PMOS, which increases efficiency of the whole system.

This configuration results to be stable and safe also when command signals on gates of MOSFETs are floating due to control unit disconnection or initialization. This is achieved by using bipolar transistors in open-collector configuration in addition with a pull-up resistor to control the switches status. It should be noted that no specific driver circuits are used.

For border cells, i.e. bottom cell 0 and top cell $N$-1, switches architecture is quite different because gate command signal of MOSFETs cannot overcome rail voltage levels. So, bottom cell has both switches made by NMOS transistors. This solution makes the cell simpler to control and improves system efficiency. On the contrary, top switches use only Pchannel transistors. In this case, the cell results harder to control. In fact, in case of control unit disconnection, terminals remain floating and both switches get opened. This required the addition of a Schottky diode at the top of pack able to provide automatic connection of cell $N-2$ to the output terminal in case of control unit disconnection.

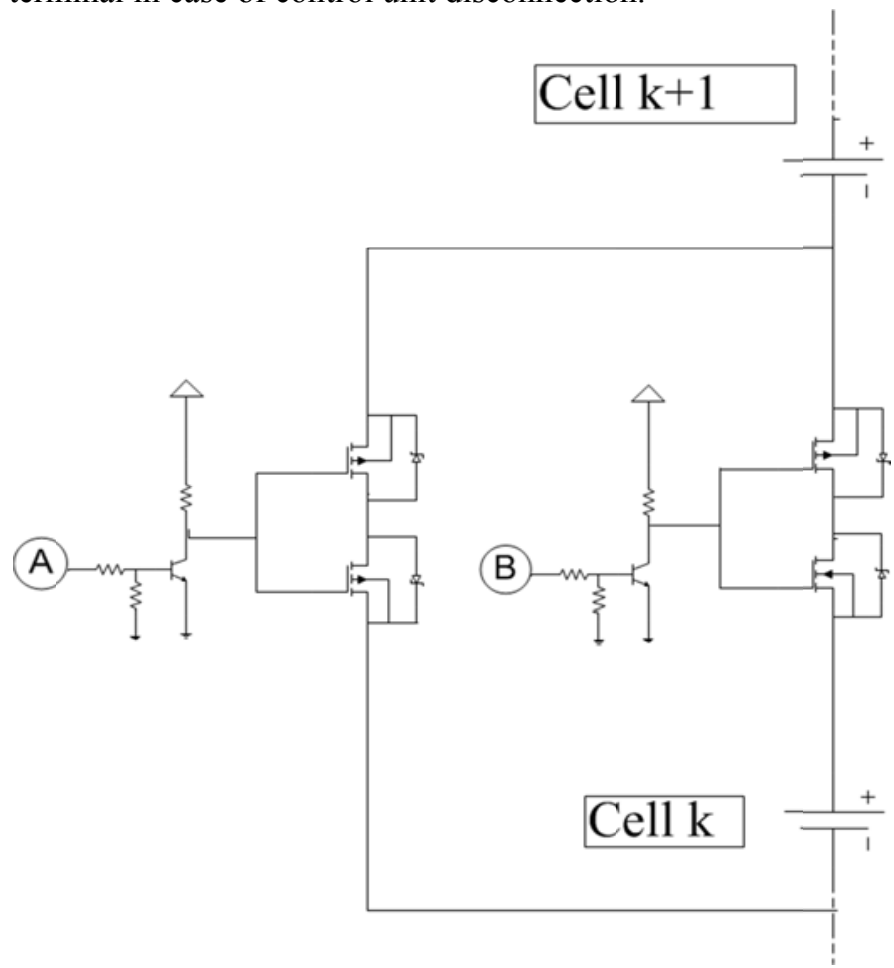

Figure 4: Simplified final schematic of a single switch block. A and $B$ are the gates for control of the switch status by the control unit.

\section{BALANCING ALGORITHM}

The algorithm that manages cells connection or bypass represents another crucial part of the system. It directly impacts performance in terms of balance effectiveness and convergence speed. The algorithm has been developed bearing in mind to achieve requested performance with the simplest algorithm architecture. The flow chart of the developed algorithm is shown inErrore. L'origine riferimento non è stata trovata.
First algorithm step is data acquisition where all needed signals are sampled (cell voltage, pack voltage, current). Core of the algorithm is the management of the cell to be disconnected, based on state of charge calculation of each battery. Several techniques for 'SoC estimation can be found in literature (V. Pop 2006), (V. Pop 2005) and (A. J. Salkind 1999). Unfortunately, most of them (e.g. OCV measurement, impedance spectroscopy, neural networks, fuzzy logic) are not suited to be used in this appllication, because some require battery disconnection or long resting times and some others need high amount of memory and computation resources. In this case the "Mixed Estimation Algorithm" (F. Codeca' Sept. 2008) has been adopted. This is the synthesis of CoulombCounting and Model-Based techniques and permits to minimize computation efforts maintaining accurate estimation results (F. Codeca' 2009) and (F. Codeca' 2009).

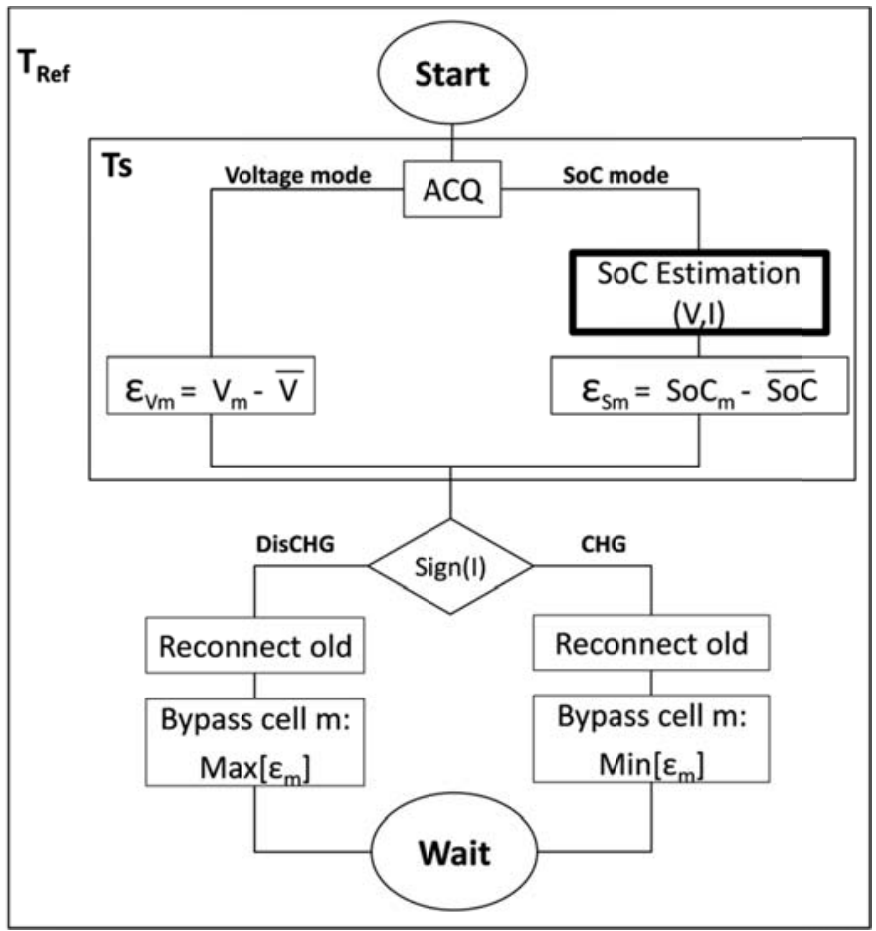

Figure 5: Developed algorithm flowchart

Here is explained the working principle of the decision strategy. As first step, the algorithm calculates the mean SoC over all the cells. Then, for each $m$-th cell it calculates the deviation $\varepsilon_{m}$ of its $\mathrm{SoC}$ value with respect to the mean one. This deviation looks like:

$$
\varepsilon_{s m}=S o C_{m}-\overline{S o C}
$$

Checking the current sign, algorithm establishes the working phase: if in discharge operation, cell with the lowest deviation is selected for disconnection, otherwise the one with the highest deviation value. The selected cell is bypassed and the previously bypassed one is re-connected into the string.

The algorithm runs cyclically. In particular, the first step and SoC estimation are performed with time interval $T s$ while pack configuration is updated with time interval Tref (refresh time). For proper work, Tref $\geq T s$. Lowering $T s$ value increases the control bandwidth to prevent cell damage caused by over-voltages and under-voltages. On the contrary, Tref is a critical parameter that hardly affects performance of the 
system and whose optimal value will be discussed in next section.

\section{PROTOTYPING}

A picture of the realized prototype is shown in Figure 6.

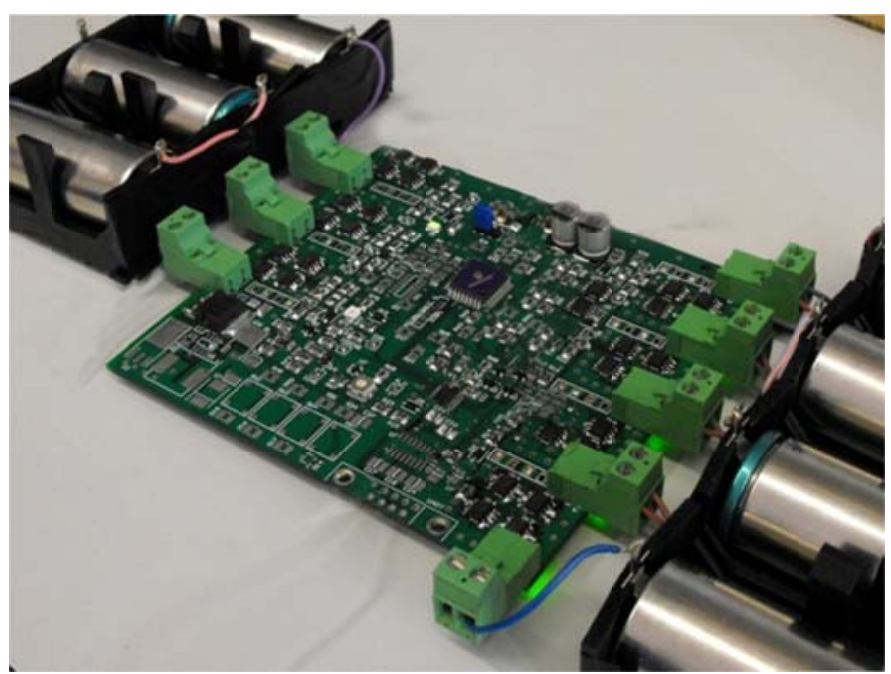

Figure 6: Picture of the circuit implementing the proposed BMS architecture.

\section{A. Refresh time calculation}

Data acquisition and SoC estimation are performed with time interval $T s$ while pack configuration is refreshed with time interval Tref. Let us focus the influence of Tref on system performance.

As Ts value doesn't impact on performance of the system as long as it remains small enough to allow an adequate acquisition rate, its value has been set constant to 2 seconds. On the contrary, Tref is a critical parameter that hardly affects performance of the system: too large Tref values correspond to a loss of balance accuracy, while too small Tref values increase the stress of system and cells (e.g. due to current spikes associated to the reconfiguration process).

An analytical estimation of the best value of Tref has been investigated. We consider a string of $N$ perfectly balanced cells discharged at $1 \mathrm{C}$. In order to compare performance obtained with different Tref values, a figure of merit $Q$ has been defined:

$$
Q:=\frac{1}{N} \sum_{m=0}^{N-1} \frac{1}{N} \int_{0}^{(N-1) T_{r e f}}\left|\mathrm{SoC}_{m}(t)-\overline{\mathrm{SoC}}\right| d t
$$

The parameter $Q$ is the integrated absolute error in $\mathrm{SoC}$, averaged across all the cells. This means that the balancing effect is much better as the $Q$ factor decreases. Figure 7 graphically represents the terms of evaluation of eq. 2 for $Q$ calculation in case of $N=7$ cells. Starting from the condition of all cells perfectly balanced with $\mathrm{SoC}=\mathrm{SoC}_{\mathrm{i}}$ and considering the discharge phase of the pack, we consecutively disconnect one cell at a time for 7 consecutive reconfigurations. Figure 7 shows the superposition of $\mathrm{SoC}$ evolutions in time of a single cell $-\mathrm{SoC}_{2}(\mathrm{t})-$, and the $\mathrm{SoC}$ mean value of the entire pack $\overline{\mathrm{SoC}}$. The horizontal step represents the transient of disconnection of the considered cell.
Numbering cells from 0 to $N-1$, SoC of each $m$-th cell depends on time as:

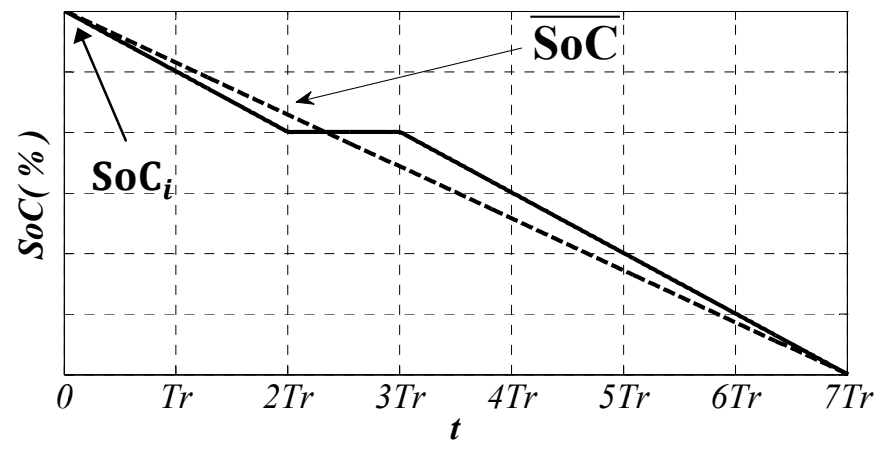

Figure 7: Trend of the SoCs during a period of seven consecutive reconfigurations. $\mathrm{Tr}$ is the refresh time and vertical axis represents SoC in arbitrary units.

$\mathrm{SoC}_{m}(t)=\left\{\begin{array}{lr}\mathrm{SoC}_{i}-t & \mathrm{t} \in T 1 \\ \mathrm{SoC}_{i}-m T_{\text {ref }} & \mathrm{t} \in T 2 \\ \mathrm{SoC}_{i}-m T_{\text {ref }}-\left(t-(m+1) T_{\text {ref }}\right) & \mathrm{t} \in T 3\end{array}\right.$

where

$$
\left\{\begin{array}{l}
T 1=0 \leq t \leq m T_{\text {ref }} \\
T 2=m T_{\text {ref }} \leq t \leq(m+1) T_{\text {ref }} \\
T 3=(m+1) T_{\text {ref }} \leq t \leq N T_{\text {ref }}
\end{array}\right.
$$

The SoC mean value trend is a straight line described by the equation

$$
\overline{\mathrm{SoC}}=\mathrm{SoC}_{i}-\left[\frac{N-1}{N}\right] t
$$

Deviation $\Delta \mathrm{SoC}_{m}(t)$ of the SoC of the $m$-th cell with respect to $\overline{\mathrm{SoC}}$ results

$$
\begin{aligned}
& \Delta \mathrm{SoC}_{m}(t) \\
&= \begin{cases}-\frac{1}{N} t & \mathrm{t} \in T 1 \\
-\frac{1}{N} m T_{\text {ref }}+\frac{N-1}{N}\left(t-m T_{\text {ref }}\right) & \mathrm{t} \in T 2 \\
\frac{N-1-m}{N} T_{\text {ref }}-\frac{1}{N}\left(t-(m+1) T_{\text {ref }}\right) & \mathrm{t} \in T 3\end{cases}
\end{aligned}
$$

and the mean value of $\Delta \mathrm{SoC}_{m}(\boldsymbol{t})$ is:

$$
\overline{\Delta \mathrm{SoC}_{m}}(t)=\frac{1}{2} \frac{m^{2}}{N(N-1)}+\frac{1}{2} \frac{(N-1-m)^{2}}{N(N-1)}
$$

Finally the value of the $Q$ factor, i.e. the mean value over all cells, is

$$
Q=\frac{1}{N} \frac{(2 N-1)}{6} T_{r e f}
$$




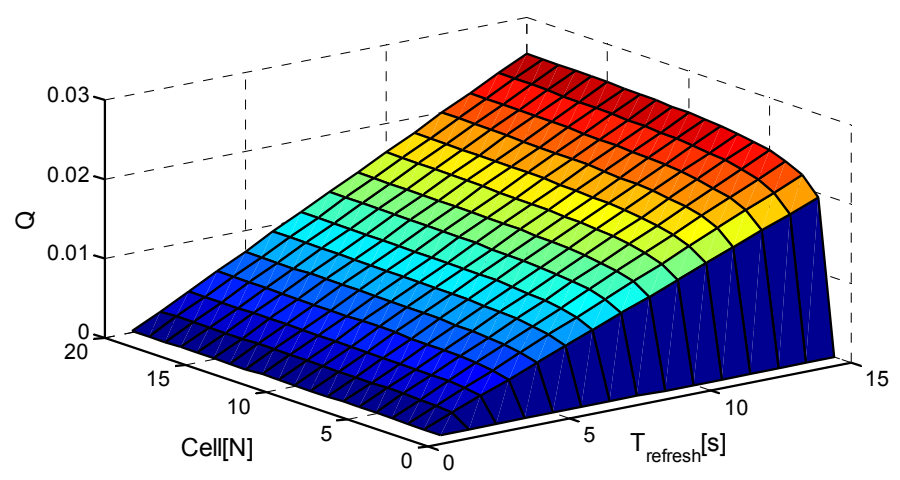

Figure 8: Quality factor $Q$ versus number of cells $(N)$ and refresh time (Tref).

The $Q$ value is directly proportional to the refresh time Tref, i.e. an increase of the refresh time worsens the balancing effect. Moreover, increasing the number $N$ of cells in the string worsens the balancing quality (even if not linearly). Figure 8 represents the trend of the quality factor $Q$ as a function of cell number $N$ and refresh time Tref.

By using the realized prototype of BMS, i.e. a string of $N=7$ cells, the parameter $Q$ has been experimentally calculated for different values of Tref. Precisely, several values of $Q$ have been measured with the same Tref and different current profiles, and their mean value has been considered as final result. Measurements confirm the dependence of $Q$ on Tref, as Table 1 summarizes.

\begin{tabular}{cc}
\hline $\boldsymbol{T}_{\boldsymbol{r e f} \boldsymbol{f}}(\mathbf{s})$ & $\boldsymbol{Q}$ \\
\hline 2 & $2.610^{-4}$ \\
5 & $1.410^{-3}$ \\
10 & $1.510^{-3}$ \\
20 & $2.210^{-3}$ \\
30 & $2.810^{-3}$ \\
60 & $4.210^{-3}$ \\
90 & $7.810^{-3}$ \\
120 & $9.510^{-3}$ \\
\hline
\end{tabular}

Table 1: Experimentally measured $Q$ factor (mean) for different values of refresh time Tref.

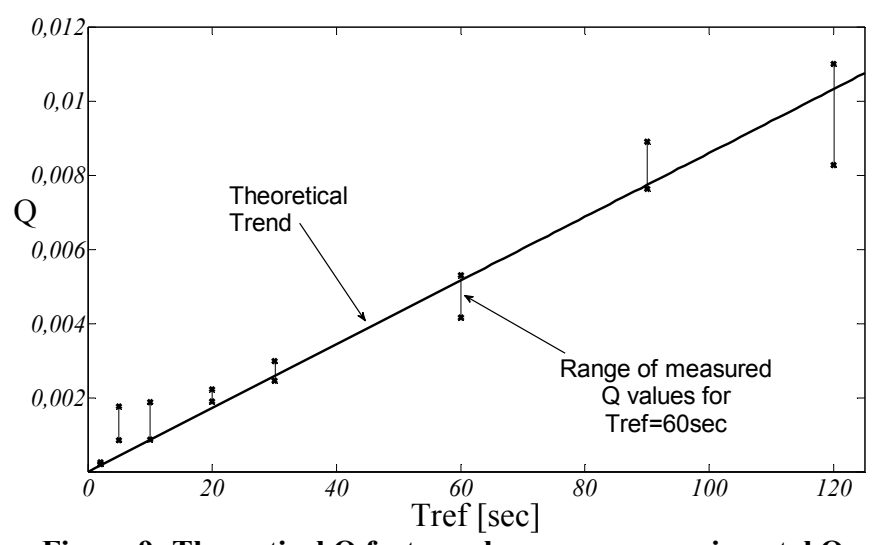

Figure 9: Theoretical $Q$ factor values versus experimental $Q$ measurements. Sets of measured $Q$ values at the same refresh time are represented with vertical bars that define their range of variation, i.e. the experimental uncertainty.

Experimental results have been compared with $Q$ values expected by (8) for $N=7$. Figure 9 compares calculated and measured $Q$ values. A good agreement is verified over all Tref considered range, both in terms of trend and variance of measured data around expected values.

In order to visually highlight the balance worsening as Tref increases, Figure 10 shows the comparison of the $\mathrm{SoC}$ of a 7cell string in case of Tref $=2 \mathrm{~s}$ and Tref $=120 \mathrm{~s}$.

\section{CONCLUSIONS}

A new BMS for string-packs of Li-ion cells has been proposed. Core of the architecture is the technique of redundant cell, i.e. the reversible disconnection of a cell in the battery pack.

Main advantages of the proposed architecture are optimal balancing of the battery pack during operation and intrinsic hardiness to cell faults. Moreover, the proposed architecture is very flexible, economical, stable and considerably spacesaving.
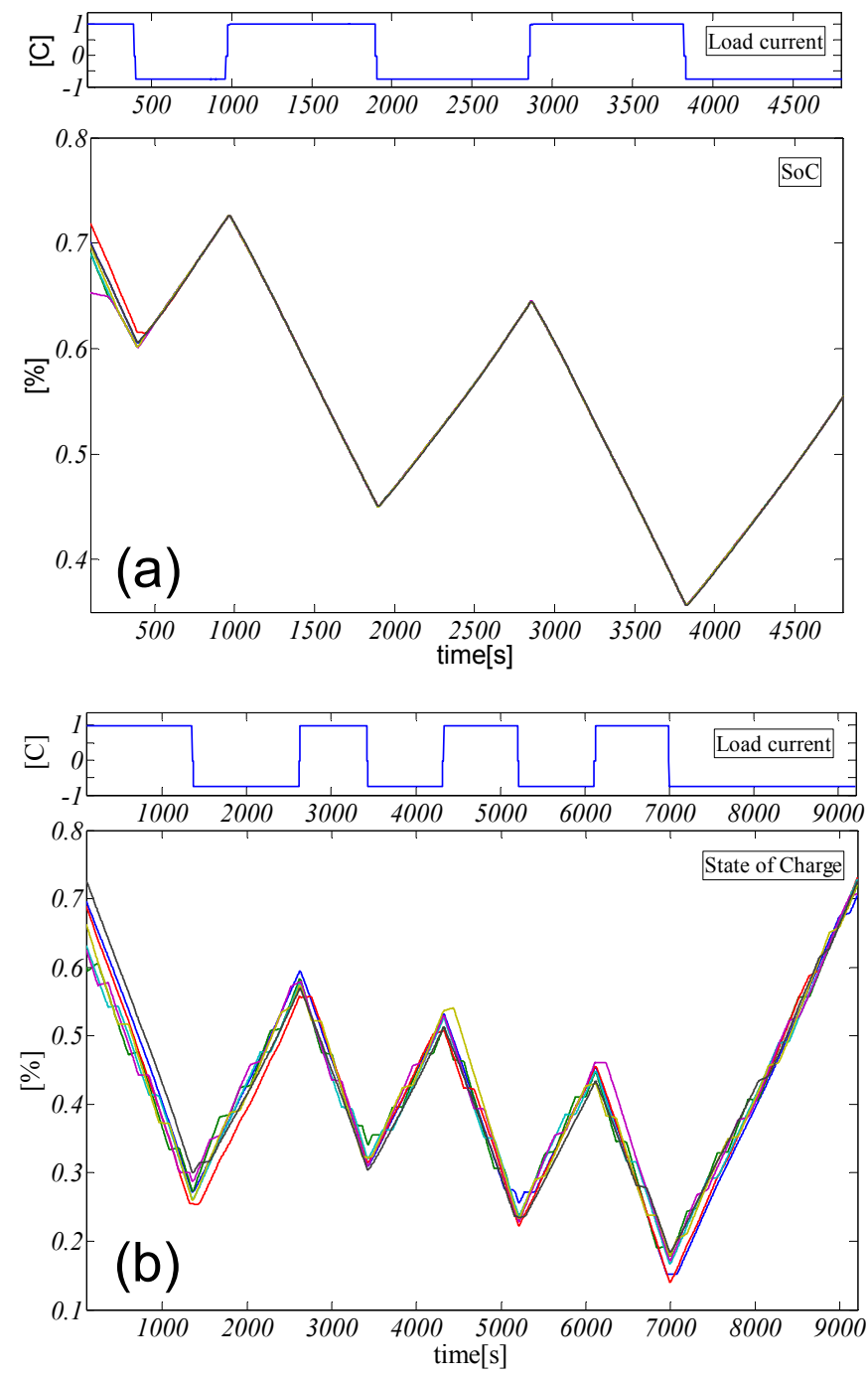

Figure 10: Comparison of the $\mathrm{SoC}$ of a 7-cell string in case of (a) Tref $=2$ sec and (b) Tref $=120$ sec. The plot shows also the considered load current profile (each line represents one of the 7 cells).

A drawback is that a substantial effectiveness of the technique limits the dimension of the pack to about 10 cells, i.e. up to $500 \mathrm{~W}$. This means that the proposed BMS architecture is suited for low-power automotive applications, 
such as electric scooters and bikes. For higher power demanding loads, a modular combination of several cellredundant BMSs can be conceived. Of course, in this case the complexity of the layout also sensibly increases.

\section{REFERENCES}

A. Emadi, YJLAKR 2008, 'Power electronics and motor drives in electric, hybrid electric, and plug-in hybrid electric vehicles', Transaction on Industrial Electronic.

A. J. Salkind, CFPSTADER 1999, 'Determination of state-ofcharge and state-of-health of batteries by fuzzy logic methodology', Journal of Power Sources, vol 80, no. 1, pp. 293-300.

Affanni, BFGT October, 2005, 'Battery Choice and Management for New-Generation Electric Vehicle', IEEE Transaction on industrial electronics.

C. S.Moo, YCHISTJCC 2003, 'Dynamic Charge Equalisation for series-connected batteries', IEE Proceedings in Electric Power Applications, Sept. 2003, pp. 501-505.

F. Codeca', VMSMS 2009, 'The mix estimation algorithm for State-of-Charge estimator Analysis of the sensitivity to measurement errors', 48th IEEE Conference on Decision and Control, Shangay, P. R. China.

F. Codeca', SMSVM 2009, 'The mix estimation algorithm for state-of-charge estimator analysis of the sensitivity to model errors.', 2nd ASME Annual Dynamic Systems and Control Conference, Hollywood, Ca, USA.

F. Codeca', SMSGR Sept. 2008, 'On battery State of Charge estimation: a new mixed algorithm', 17th IEEE International conference on Control Applications, Part of 2008 IEEE Multi-conference on systems and control, San Antonio, Texas, USA.

Ferdowsi, ABAM 2005, 'Battery charge equalization-state of the', Proc. Future Transport Technology Conference, pp. $905-910$.

H. Sakamoto, KM,KNKHSTKYGA 1998, 'Balanced charging of series connected battery cells', Twentieth International Telecommunications Energy Conference, INTELEC, San Francisco CA, USA.

H.V. Venkatasetty, YUJ 2002, 'Recent advances in Lithiumion and Lithium-Polymer batteries', Proc. 17th annual battery conf. Applications and advances, pp. 173-178.

Hong-Sun Park, C-EKC-HKG-WMJ-HL 2009, 'A Modularized Charge Equalizer for an HEV LithiumIon Battery String Low Voltage Stress for HEV Lithium-ion Battery String', IEEE Transaction on Industrial Electronics, vol 56, no. 5, pp. 1464-1476.

J. Chatzakis, KK,NCVSNM 2003, 'Designing a new generalized battery management system', IEEE Transaction on Industrial Electronic, vol 50, no. 5, pp. 990-999.

Marc W. Yuzcow, STM 1997, 'Design consideration for Lithium-ion cells. Part II: Safety and abuse testing', IEEE Transaction on Industry applications.

N. H. Kutkut, HLNWDMD 1999, 'Design considerations for charge equalization of an electric vehicle battery system', IEEE Transaction on industry applications, pp. 28-35.

P. Casasso, AFGGPGFVGF 2003, 'Feasibility, test and novel design of battery packs for EV Scooter', The 29th
Annual Conference of the IEEE Industrial Electronics Society, IECON '03..

S. Rodrigues, NMAS 1999, 'A review of state of charge indication af batteries by means of a.c. impedance measurements', Journal of Power Sources, no. 87, pp. 12-20.

Shneider, SWMAPJ 2001, 'A review of cell equalization methods for lithium-ion and lithium polymer battery systems', Proc. SAE World Congress.

V. Pop, HJBPHLNAPPLR 2005, 'State-of-the-art of battery state-of-charge determination', Measurement Science and Technology, no. 16, pp. 93-110.

V. Pop, HJBJHGOHVPPLRDDPHLN 2006, 'Modelling battery behavior for accurate State-of-charge indication', Jurnal of the Electrochemical Society, vol 11, no. 153, pp. A2013-A2022. 